\title{
Different Channel Estimation and ICI Cancellation Techniques in MIMO-OFDM Wireless Communication Systems
}

\author{
Amit Kapoor ${ }^{1}$, Ishan Khurana ${ }^{2}$ \\ ${ }^{l}$ Department of Electronics and Communication, Lovely Professional University, Phagwara, Punjab, India \\ ${ }^{2}$ Department of Electronics and Communication, Lovely Professional University, Phagwara, Punjab, India
}

\begin{abstract}
MIMO OFDM is one of the prominence communication schemes with multi-carrier modulation. The MIMO combined with OFDM modulation technique provides the reliable high data rate transmission over the broadband wireless channels. They have been widely studied and employed for $4 G$ systems such as Wi-Fi, DVBT, Wi-MAX and LTE-A. The major challenge in MIMO-OFDM systems is how to estimate the channel. The estimation of the channel is done by various algorithms such as Least Square (LS) and Minimum Mean Square Error (MMSE). The main issue in OFDM systems is the problem of ICI (Inter carrier Interference) which is due to the loss of the orthogonality between the sub-carriers and the signal transmitted.ICI self-cancellation is one of the reduction methods to reduce the ICI effect in the signal.
\end{abstract}

Keywords: ICI, LS, MIMO, MMSE, OFDM

\section{Introduction}

The key challenge nowadays in wireless communication is to increase the data rate with improving the transmission reliability of the data. Multiple input and multiple output wireless technology can meet these demands by offering increased spectral efficiency through spatial multiplexing and improved reliability with the help of antenna diversity gain. The first product of MIMO technology have become available which is the preIEEE 802.11n wireless local area network (WLAN) systems by Atheros Communication Inc., Broadcom Corporation, Marvell Semiconductor, Inc.Current industry trends suggest that large-scale deployment of MIMO wireless systems will initially be seen in WLANs and in wireless metropolitan area networks (WMANs). Corresponding standards currently under definition include the IEEE 802.11n WLAN and IEEE 802.16 WMAN standards. Both standards define air interfaces that are based on the combination of MIMO with Orthogonal frequency division multiplexing (OFDM) modulation (MIMO-OFDM).

\subsection{MIMO}

MIMO (Multiple Input Multiple Output) is a wireless technology that uses multiple transmitters and receivers to transfer more than one data at the same time in the same frequency band. MIMO technology takes the advantage of the radio wave system called multipath. In multipath, the transmitted information reflected from walls, ceilings, and other objects and reached the receiving antenna multiple times via different angles and at slightly different timing. For multipath signals travelling through different paths has their independent effect imposed by the channels which are path gain or loss, path frequency offset, change in angle and path time delay. MIMO is a form of spatial diversity technique [1].By using multiple antennas at the transmitter and the receiver side which means multiple RF chain will increase the cost and the complexity of the system. This problem can be overcome by using a new technique called antenna selection technique which reduces the complexity of the system. This antenna selection technique work good for the uncorrelated MIMO channels in the system. However, practically cellular channels exhibit fading correlation due to the lack of spacing between the antennas [2].

As a result of using the MIMO system, the performance benefits are due to the spatial multiplexing gain, diversity gain and the array gain [3]

\subsection{OFDM}

Orthogonal frequency division multiplexing is one of the multi-carrier modulation techniques that transmit signals through multiple carriers. These carriers (sub carriers) have different frequencies and they are orthogonal to each other. Basically OFDM is a combination of modulation and multiplexing.OFDM is an alternative wireless modulation technology to CDMA. OFDM is a modulation scheme that allows digital data to be efficiently and reliably transmitted over a radio channel even in multipath environments [2]. 


\subsection{PROBLEMS IN OFDM}

\subsubsection{Synchronization}

It is one of the crucial problems in the receiver side that to sample the incoming signal correctly. If the wrong sequence of input signal is processed, then the FFT will not recover the original data correctly on the carrier. There is a need of timing lock which is the time shift theorem. To avoid this type of problem more than one complete sequence of time sequence should be transmit in order to increase the tolerance.

\subsubsection{Phase Noise}

The phase noise is added to the receiver side by the local oscillator. Phase noise has two types of effects which are Common Phase Error and Inter Carrier Interference. ICI is difficult to overcome because of the additive noise which is separate from other carriers.

\subsubsection{Inter Carrier Interference (ICI)}

The main issue in OFDM system is the problem of ICI which is due to the loss of orthogonality between the sub-carriers and the signals transmitted on each carrier which are not independent of each other.

\section{Inter-Carrier Interference (Ici) Mechanism Of Ofdm Systems}

A well-known problem of OFDM system is its sensitivity to frequency offset between the transmitted and received signal which is caused by Doppler shift in the channel. This carrier frequency offset causes loss of orthogonality between sub-carriers and the signals transmitted on each carrier which are not independent of each other. This leads to the inter-carrier interference (ICI) in OFDM. Researchers have proposed various methods to overcome the ICI in OFDM systems. The previously approaches that have been used to reduce ICI are categorized as frequency-domain equalization, time-domain windowing, and the ICI self-cancellation (SC) scheme [5]. In this report, the effects of ICI have been analyzed and three solutions to overcome the ICI have been presented which are as follows:

a. The first method to overcome the ICI is a self-cancellation scheme, in which redundant data is transmitted onto adjacent sub-carriers such that the ICI between adjacent sub-carriers cancels out each other at the receiver end.

b. The second method is the windowing technique.

c. The other techniques which are Maximum Likelihood (ML) estimation and the Extended Kalman Filter (EKF) method statistically estimate the frequency offset and correct the offset using the estimated value at the receiver.

\subsection{Self-Cancellation Method}

ICI self-cancellation is a scheme that was introduced by Zhao and Sven-Gustav in 2001 to combat and suppress ICI in OFDM system [6]. In ICI, the main idea is mapping the input data symbol onto a group of subcarriers with predefined coefficients such that theICI signals which are generated within that group should cancel each other. The basis of ICI self-cancellation is that the difference between the ICI co-efficient of two consecutive sub-carriers are very small.In this method, one data symbol is modulated into two sub-carrier. Let us suppose that the data symbol ' $a$ 'is modulated in to the 1st sub-carrier then '- $a$ ' is modulated in to the 2nd subcarrier. Hence the ICI generated between the two sub-carriers almost mutually cancels each other. This method is suitable for multipath fading channels as here no channel estimation is required because in multipath channel estimation fails as the channel changes randomly. This method is also suitable for flat channels. The method is simple, less complex and effective. The major drawback of this method is the reduction in band width efficiency as same symbol occupies two subcarrier.

\subsection{Windowing Technique}

We know that OFDM signal has widely spread power spectrum. So if this signal is transmitted in a band limited channel, certain portion of the signal spectrum will be cut off, which will lead to inter carrier interference. To diminish the interference the spectrum of the signal wave form need to be more concentrated. This is achieved by windowing the signal. Basically windowing is the process of multiplying a suitable function to the transmitted signal wave form. The same window is used in the receiver side to get back the original signal. The ICI will be eliminated if the product of the window functions satisfies the Nyquist vestigial symmetry criterion.

The conventional frame-by-frame time limited orthogonal multicarrier signal $\mathrm{S}(\mathrm{t})$ can be expressed as:

$s(t)=\sum_{k=-\infty}^{\infty} \sum_{n=0}^{N-1} w(t-k T) a_{n, k} \exp \left(j n \omega_{\Delta}(t-k T)\right)$ 
In the above equation, $\left\{a_{n, k}\right\}$ is a complex sequence, $n w_{\Delta}\{n=0,1, \ldots \ldots, N-1\}$ are carrier frequencies and theyare equally spaced with $w_{\Delta}$. The window function $\mathrm{w}(\mathrm{t})$ has a length of $\mathrm{T}$ and it modifies the waveform of the multicarrier signal in each frame. The ICI for this multicarrier signal can be avoided if the window function $\mathrm{w}(\mathrm{t})$ and the carrier separation $n w_{\Delta}$ are correctly chosen. In order to provide a matched receiver for the transmitted signal, the window functions in both the transmitter and the receiver are selected to be equal. The ICI can be determined by examining the cross correlation between two carriers of the transmitted signals. For a complex sequence $\left\{a_{n, k}\right\}$, the ICI is given by:

$a_{n, k} a_{m, k}^{*} \int_{-T / 2}^{T / 2} w^{2}(t) \exp \left(-j \omega_{\Delta}(n-m) t\right) d t=0$

\subsection{Maximum Likelihood Estimation}

Another method for frequency offset correction i.e. ML estimation in OFDM systems was suggested by Moose. In this approach, the frequency offset is first statistically estimated using a maximum likelihood algorithm and then cancelled at the receiver. This technique involves the replication of an OFDM symbol before transmission and comparison of the phases of each of the subcarriers between the successive symbols. When an OFDM symbol of sequence length is replicated, the receiver receives, in the absence of noise, the $2 \mathrm{~N}$ point sequence $r(n)$ is given by:

$k=0,1,2, \ldots \ldots . . N-1, N \geq 2 K+1$

In the above equation, $X(k)$ are the $2 k+1$ complex modulation values used to modulate $2 k+1$ subcarriers, $H(k)$ is the channel transfer function for the $k$ th carrier and $\mathrm{E}$ is the normalized frequency offset of the channel. The first set of $\mathrm{N}$ symbols is demodulated using an N-point FFT to yield the sequence $R_{1}(k)$, and the second set is demodulated using an N-point FFT to yield the sequence $R_{2}(k)$. The frequency offset is the phaise difference between $R_{1}(k)$,and $R_{2}(k)$, that is $R_{2}(k)=R_{1}(k) e^{j 2 \pi \varepsilon}$

Adding the AWGN yields

$Y_{1}(k)=R_{1}(k)+W_{1}(k)$

$Y_{2}(k)=R_{1}(k) e^{j 2 \pi \varepsilon}+W_{2}(k)$

$k=0,1,2 \ldots \ldots \ldots N-1$

The maximum likelihood estimate of the normalized frequency offset is given by:

$\hat{\varepsilon}=\left(\frac{1}{2 \pi}\right) \tan ^{-1}\left\{\frac{\sum_{k=-K}^{K} \operatorname{lm}\left[Y_{2}(k) Y_{1}^{*}(k)\right]}{\sum_{k=-K}^{K} \operatorname{Re}\left[Y_{2}(k) Y_{1}^{*}(k)\right]}\right\}$

The main disadvantage of OFDM is its susceptibility to small differences in frequency at the transmitter and receiver which the frequency offset. The frequency offset can be caused by Doppler shift due to relative motion between the transmitter and receiver, or by differences between the frequencies of the local oscillators at the transmitter and receiver [4]. The frequency offset is modulated as a multiplicative factor introduced in the channel, as shown below:

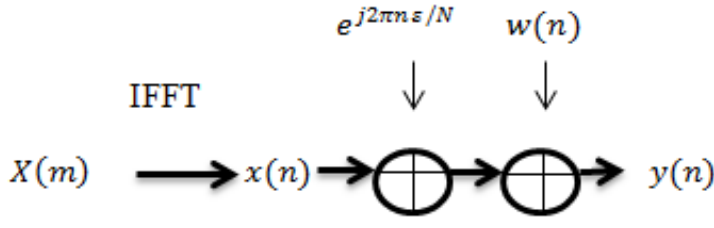

The received signal is given by:

$y(n)=x(n) e^{\frac{j 2 \pi n \varepsilon}{N}}+w(n)(1.05)$

Here $\varepsilon$ is the normalized frequency offset, and is given by $\Delta f N T_{s} . \Delta f$ is the frequency difference between the transmitted and received carrier frequencies and $T_{s}$ is the sub-carrier symbol period. $w(n)$ is the AWGN introduced in the channel. ICI self-cancellation is a scheme that was introduced by Zhao and Sven-Gustav Hageman in 2001 into combats and suppresses ICI in OFDM [6]. The main idea of OFDM system is to modulate the input data symbol onto a group of subcarriers with predefined coefficients such that the generated ICI within the group will cancel each other. 


\section{Mimo Ofdm System Model}

The Block diagram of MIMO-OFDM is shown in fig. 1.At the transmitter side, a serial data bit stream is mapped to a symbol stream by a modulator. Then, this serial symbol stream is converted into parallel substreams. Next, pilot symbols for the channel estimation are inserted into these parallel sub-streams, in the frequency-domain, prior to the OFDM modulation. The OFDM modulation is then implemented by performing the inverse discrete Fourier transform (IDFT). Each transmits antenna sends independent OFDM symbols. After this cyclic prefix is added in order to reduce the effect of ISI. After that bits are converted from parallel to serial and given to the antenna. At the receiver side the bits are converted from serial to parallel after getting from the antenna. Then cyclic prefix is removed which is added at the transmitter side and then OFDM mod again is performed in which DFT is performed. After this the channel estimation block comes in order to estimate the channel accurately. At last demapping is done in order to get the desired output.

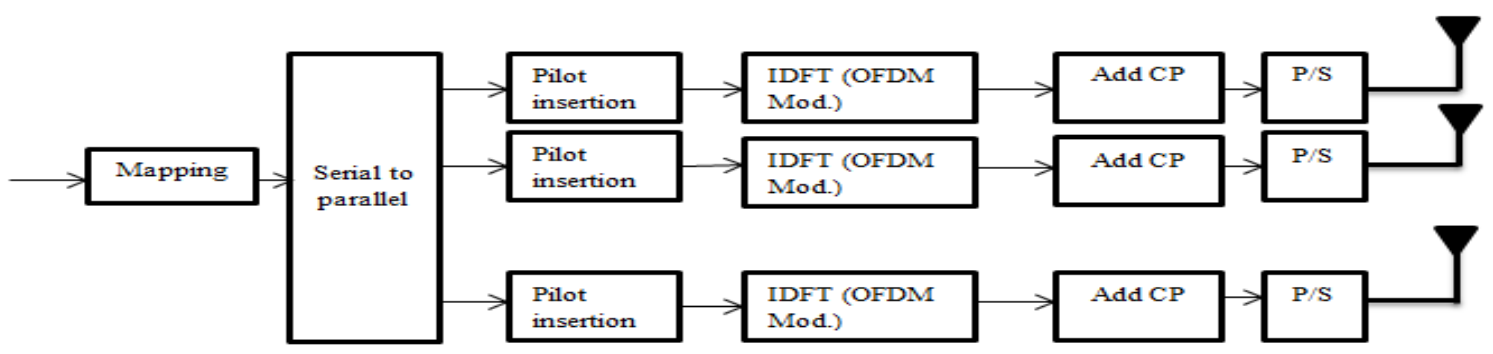

(a)

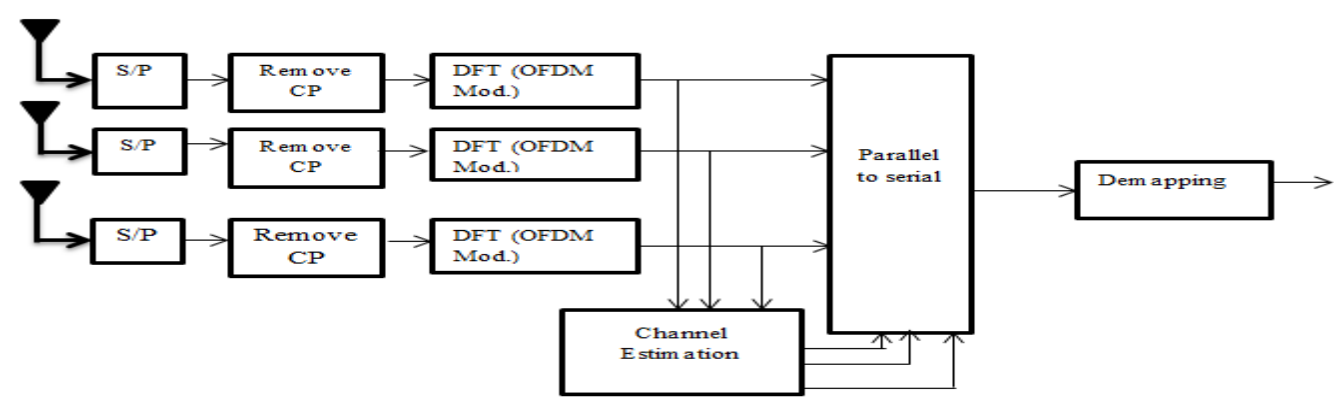

(b)

Fig. 1 Block Diagram of MIMO-OFDM system, (a) Transmitter, (b) Receiver

\section{Channel Estimation}

Channel estimation plays an important role in MIMO-OFDM systems. It is an important part of communication system. There are number of channel estimation methods which have already been proposed for MIMO-OFDM systems. A number of channel estimation methods have been proposed for MIMO-OFDM systems which can be broadly classified into three classes: the training based method, the blind method and semi blind method (combination of the first two methods).

Channel estimation over rapidly time varying multipath fading channels has been considered in the papers [8]-[9]. The Doppler spread information is also utilized for computing the frequency and time domain channel correlations in the channel estimation process. The present channel estimation methods are divided into two types: based on the pilots and the second is the blind channel estimation which does not use pilots. Blind channel estimation methods do not use pilots and have higher spectral efficiency. Blind channel estimation methods are not suitable for applications with fast varying fading channels. The channel estimation methods which are widely used for the pilot aided channel estimation methods are divided into two types: the block type pilot channel estimation and the comb type pilot channel estimation [7].

In the block type pilot channel estimation, pilots are inserted into all the subcarriers of one OFDM symbol with a certain period and they can be adopted in slow fading channel which means the channel is static within a certain period of OFDM symbols. The comb-type refers to the pilots which are inserted at some specific subcarriers in each OFDM symbol. The comb-type is preferred in fast varying fading channel that is the channel varies over two adjacent OFDM symbols but remains static with one OFDM symbol. When the fading channel cannot be viewed as a static within an OFDM symbol, then ICI occurs whereas the comb-type pilot patterns cannot eliminate ICI [7]. There are some channel estimation methods for the pilot aided channel estimation which are LS (Least Square) and MMSE (Minimum Mean Square Estimation) [8]. 


\subsection{LS (Least Square) ESTIMATOR}

The channel frequency response at pilot subcarriers is assumed to be:

$H_{p}=\left[\begin{array}{llll}H_{p}(0) & H_{p}(1) \ldots . . & H_{p}\left(N_{p}-1\right)\end{array}\right]^{T}$

In the above equation, $N_{P}$ is the number of pilots. The extracted pilot signal vector is given by:

$Y_{p}=\left[\begin{array}{llll}Y_{p}(0) & Y_{p}(1) \ldots . . & Y_{p}\left(N_{p}-1\right)\end{array}\right]^{T}$

After that the vector $Y_{p}$ can be further given as:

$Y_{p}=X_{p} H_{p}+W_{p}$

In the above equation, $W_{p}$ is the Gaussian noise vector at the pilot position and it is given by:

$W_{p}=\left[\begin{array}{llll}W_{p}(0) & W_{p}(1) \ldots . . & W_{p}\left(N_{p}-1\right)\end{array}\right]^{T}$

Let the estimated channel response is given by:

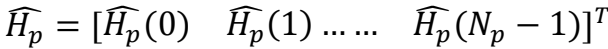

According to the LS criterion, $e_{p}=\left(Y_{p}-H_{p} X_{p}\right)^{T}\left(Y_{p}-H_{p} X_{p}\right)$ should be minimum.

\subsection{MMSE (Minimum Mean Square Error) ESTIMATOR}

Minimum mean square error (MMSE) estimator has better estimation accuracy as compared to the LS estimator in terms of MSE. At the same MSE, the MMSE estimator has about 5 to $12 \mathrm{~dB}$ SNR gain as compared to that of the LS estimator. The main issues of MMSE estimator is the higher computation complexity. With the increase in the number subcarriers, its complexity will be increased exponentially. The MMSE estimation in frequency domain for the pilot subcarriers is given by:

$\widehat{H_{p, m m s e}}=R_{H_{p} H_{p, l s}} R_{H_{p} H_{p, l s}}^{-1} \widehat{H_{p, l s}}=R_{H_{p} H_{p}}\left(R_{H_{p} H_{p}}+\sigma_{w}^{2}\left(X_{p} X_{p}^{H}\right)^{-1}\right)^{-1} \widehat{H_{p, l s}}$

In the above equation $\overline{H_{p, l s}}$ is the channel frequency response at pilot positions using LS algorithm and $\sigma_{w}^{2}$ is the variance of the AWGN noise [8].

\section{Conclusion}

The demand for high data rate wireless communication has been increasing rapidly over the last decade. MIMO OFDM is one of the emerging communication schemes that provide efficient communication with Multi-Carrier Modulation. In this paper I have discussed about the basic idea behind the MIMO-OFDM and the different techniques of remove the ICI in OFDM and different channel estimation methods in order to estimate the channel correctly.Researchers have proposed various methods to combat the ICI in OFDM systems. Self-cancellation technique is mostly used to mitigate the ICI effect in OFDM systems. The major challenge is to estimate the channel in MIMO-OFDM. In addition, statistical approaches have also been explored to estimate and cancel ICI. Kalman Filtering is an effective method to filter impurities in linear systems. The kalman filter basically consists of a set of mathematical equations that provides an efficient computational means to estimate the state of a process that minimizes the mean of the squared error.

\section{References}

[1.] TheodoreS.Rappaport, "Wireless Communication:Principles andPractice", Prentice-Hall, 2 Edition, 2010, India.

[2.] Yong Soo Cho, Jaekwon Kim Won Young Yang, Chung-Gu-Kang, "MIMO-OFDM Wireless Communication with MATLAB", John Willey \& Sons (Asia) Pte Ltd.

[3.] A.J. Paulraj, D. A. Gore, R.U. Nabar, H. Bolcskei, "An overview of MIMO communications - a key to gigabit wireless," Proceedings of the IEEE, vol.92,pp. 198-218, Feb. 2004.

[4.] P. H. Moose, A technique for orthogonal frequency division multiplexing frequency offset correction, IEEE Trans. Commun., vol. 42, pp. 2908-2914, 1994.

[5.] J. Armstrong(1999), "Analysis of new and existing methods of reducing intercarrier interference due to carrier frequency offset in OFDM,” IEEE Transactions on Communications, vol. 47, pp. 365 - 369, March 1999.

[6.] Zhao and S. Haggman (2001), "Intercarrier interference self cancellation scheme for OFDM mobile communication systems," IEEE Transactions on Communications, vol. 49, pp. 1185 - 1191, July 2001.

[7.] K.Vidhya and R. Shankar kumar (2012), "Channel Estimation Techniques for OFDM Systems", Proceedings of the International Conference on Pattern Recognition, Informatics and Medical Engineering, March 21-23, 2012.

[8.] K.VidhyaR.Shankarkumar, "Channel Estimation Techniques for OFDM Systems", Proceedings of the International Conference onPattern Recognition, Informatics and Medical Engineering, March 21-23, 2012.

[9.] A.Stamoulis, S. Diggavi, and N. Al-dhahir, "Intercarrier interference in MIMO OFDM," IEEE Trans. on Signal Processing, vol. 50, pp.2451-2464, Oct. 2002. 